\title{
Об изменении кривизны конформно-плоской метрики при преобразовании Лежандра*
}

\author{
М.В. Куркина
}

Югорский государственный университет (Ханты-Мансийск, Россия)

\section{On a Change in the Curvature of a Conformally Flat Metric under the Legendre Transformation}

\author{
M.V. Kurkina \\ Ugra State University (Khanty-Mansiysk, Russia)
}

\begin{abstract}
Известно, что теория конформно-плоских римановых метрик тесно связана с псевдоевклидовой геометрией, что обусловлено существованием канонического изометрического вложения конформно-плоской метрики в изотропный конус псевдоевклидова пространства. Впервые этот факт был замечен Х. Бринкманном, а позднее использован в работах Н. Кюипера. Геометрия однородных римановых многообразий с конформноплоской римановой метрикой изучалась в работах А.Д. Алексеевского и Б.Н. Кимельфельда, в которых дана их классификация. В неоднородном случае подобной классификации не существует, поэтому при исследовании конформноплоских римановых многообразий используются ограничения различного типа: либо на размерность многообразия, либо на топологическое строение, либо на различные типы кривизны риманового многообразия с конформно-плоской метрикой. В последнем случае хорошо известны теоремы об однородных римановых многообразиях с конформно-плоской метрикой ограниченной одномерной кривизны, полученные В.В. Славским и Е.Д. Родионовым. В данной работе исследуется поведение одномерной кривизны и кривизны Риччи при преобразовании Лежандра конформноплоской римановой метрики.
\end{abstract}

Ключевъе слова: конформно-плоские метрики, преобразование Лежандра, одномерная кривизна.

DOI 10.14258/izvasu(2018)4-16

1. Введение. Теория конформно-плоских римановых метрик тесно связана с псевдоевклидовой геометрией, что обусловлено существованием канонического изометрического вложения конформно-плоской метрики в изотропный конус псевдоевклидова пространства (подробнее

\footnotetext{
* Работа выполнена при финансовой поддержке Российского фонда фундаментальных исследований (код проектов 18-47-860016, 18-01-00620), при поддержке Научного фонда ЮГУ № 13-01-20/10.
}

It is known that the theory of conformally flat Riemannian metric is closely associated with pseudo-Euclidean geometry, due to the existence of the canonical isometric embedding conformally flat metric in pseudo-isotropic cone space. This fact was first noticed by $H$. Brinkmann, and later was used in the works of N. Kuyper. The geometry of homogeneous Riemannian manifolds with a conformally flat Riemannian metric was studied in the papers of A.D. Alekseevsky and B.N. Kimel'feld, in which their classification was given. In the inhomogeneous case such a classification does not exist, therefore, in the study of conformally flat Riemannian manifolds restrictions of various types are used: either on the dimension of the manifold, or on a topological structure, or on different types of Riemannian manifold curvatures of the conformally flat metric. In the last case, theorems on homogeneous Riemannian manifolds with a conformally flat metric of bounded one-dimensional curvature are well known, which were obtained by V.V. Slavsky and E.D. Rodionov. In this paper, we study the behavior of a one-dimensional curvature and the Ricci curvature under Legendre Transform of the conformally flat Riemannian metric.

Key words: conformal-flat metrics, Legendre transform, one-dimensional curvature.

см. [1] - [18]). Впервые этот факт был замечен H.W. Brinkmann [1], а наиболее эффектно был использован в работах N.H. Kuiper [2,3].

Конформно-плоская метрика имеет вид: $d s^{2}=$ $\frac{d x^{2}}{f^{2}(x)}$, где $f(x)$ - функция, определенная на подмножестве $D$ евклидова пространства. В данной работе будут рассмотрены только два варианта задания конформно-плоской метрики:

1. Сферическая модель; множество $D-n$ мерная сфера евклидова пространства $R^{n+1}$, 
а функция $f(x)$ сужение на сферу однородной порядка один функции $f: R^{n+1} \mapsto R$.

2. Плоская модель; множество $D=R^{n}-\mathrm{n}$ мерное евклидово пространство, а функция $f(x)$ произвольная функция на $D$.

В статье рассматривается подробно случай, когда размерность конформно-плоской метрики $n \geq 3$, в случае $n=2$ преобразование Лежандра тоже можно формально определить, но такие локальные характеристики, как кривизны полярной метрики, не определяются однозначно самой метрикой $d s^{2}$, а зависят еще от изометрического вложения в изотропный конус псевдоевклидова пространства.

2. Полярное преобразование конформноплоской метрики. В данной части мы используем обозначения и результаты работы [18]. Пусть $R$ - числовая прямая, $R^{n+1}$ - евклидово $(n+1)$-мерное арифметическое пространство, $M^{n+2}=R^{n+1} \times R$ - псевдоевклидово пространство, скалярный квадрат вектора $\vec{w}=[\vec{x}, \zeta] \in$ $M^{n+2} \quad$ в котором равен $\langle\vec{w}\rangle^{2}=|\vec{x}|^{2}-\zeta^{2}, \quad$ где $|\vec{x}|^{2}-$ скалярный квадрат вектора $\vec{x} \in R^{n+1}$. Обозначим через

$$
C^{+}=\left\{[\vec{x}, \zeta] \in M^{n+2}:|\vec{x}|^{2}-\zeta^{2}=0, \zeta>0\right\},
$$

верхнюю часть изотропного конуса в $M^{n+2}$. В дальнейшем, если будет ясно из контекста, мы станем обозначать $\vec{x}$ через $x$.

Лемма 1. Пусть на единичной сфере $S^{n} \subset$ $\mathbb{R}^{n+1}$ задана конформно-плоская метрика

$$
d s^{2}=\frac{d x^{2}}{f^{2}(x)}, \quad x \in S^{n} \subseteq \mathbb{R}^{n+1},
$$

где $f(x)$ - функция класса $C^{1}$. Тогда определено каноническое изометрическое вложение, задаваемое формулой

$$
Z: x \in S^{n} \rightarrow\left[\frac{x}{f(x)}, \frac{1}{f(x)}\right] \in C^{+} .
$$

Образ $Z\left(S^{n}\right)=F \subseteq C^{+}$- пространственноподобная $n$-мерная поверхность. В дальнейшем будем отождествлять конформно-плоскую метрику с поверхностью $F$. Предположим, что функция $f(x)$ достаточно гладкая, тогда поверхность $F$ регулярна и в каждой точке $Z(x) \in F$ определено касательное $n$-мерное пространство $T_{x}(F)$. Существует единственный вектор $Z^{*}(x) \in C^{+}$такой, что

$$
\left\langle Z, Z^{*}\right\rangle=-1, \quad Z^{*} \perp T_{x}(F),
$$

где ортогональность понимается относительно скалярного произведения в $M^{n+2}$.

Лемма 2. Пусть функция $f(\vec{x})$, задающая конформно-плоскую метрику, по однородности распространена на все пространство $\mathbb{R}^{n+1}$. Тогда вектор $Z^{*}$ явно выражается через $f$ и $\vec{\nabla} f$ в $R^{n+1}$ :

$$
Z^{*}(\vec{x})=\left[-\vec{\nabla} f+\frac{|\vec{\nabla} f|^{2}}{2 f} \vec{x}, \frac{|\vec{\nabla} f|^{2}}{2 f}\right],
$$

где $\vec{x} \in S^{n} \subset \mathbb{R}^{n+1}, \vec{\nabla} f$ градиент функции $f$ в пространстве $\mathbb{R}^{n+1}$.

Определение 1. Если точка $Z \in F$ пробегает поверхность $F$, то точка $Z^{*}$ пробегает двойственную поверхность $F^{*}$. Соответствующую конформно-плоскую метрику $d s^{* 2}=\frac{d y^{2}}{f^{* 2}(y)}, y \in$ $S^{n}$, будем называть полярной к исходной метрике $[9,11]$. Сравнивая формулы (1) и (3), имеем:

$$
\left[-\vec{\nabla} f+\frac{|\nabla f|^{2}}{2 f} \vec{x}, \frac{|\nabla f|^{2}}{2 f}\right] \equiv\left[\frac{y}{f^{*}(y)}, \frac{1}{f^{*}(y)}\right] .
$$

Откуда получаем формулы для перехода к полярной конформно-плоской метрике:

$$
f^{*}(y)=\frac{2 f(x)}{|\nabla f|^{2}}, \quad \vec{y}=\vec{x}-2 f(x) \frac{\vec{\nabla} f}{|\nabla f|^{2}} .
$$

Лемма 3. Пусть $f: R^{n+1} \rightarrow R$ произвольная однородная степени один функция на $R^{n+1}$. Отображение $H_{f}: S^{n} \rightarrow S^{n}$, определяемое формулой:

$$
H_{f}: \vec{x} \in S^{n} \rightarrow \vec{x}-2 f(x) \frac{\vec{\nabla} f}{|\nabla f|^{2}} \in S^{n},
$$

сохраняет норму вектора: $\left|H_{f}(\vec{x})\right|=|\vec{x}|$.

Определение 2. Отображение $H_{f}$ назовем конформным градиентом функции $f$. Если отображение $H_{f}$ имеет обратное $H_{f}^{-1}$, то полярная метрика определяется функцией:

$$
f^{*}(y)=\left.\frac{2 f(x)}{|\nabla f|^{2}}\right|_{x=H_{f}^{-1}(y)} .
$$

Замечание. Из определения (1) следует двойственность метрики $d s^{2}=\frac{d x^{2}}{f^{2}(x)}, x \in S^{n}$ и метрики $d s^{* 2}=\frac{d y^{2}}{f^{* 2}(y)}, y \in S^{n}$. Поэтому при наличии соответствующей регулярности функции $f^{*}(y)$ будут справедливы равенства:

$$
\begin{aligned}
& f^{*}(y)=\frac{2 f(x)}{|\nabla f(x)|^{2}}, \quad \vec{y}=\vec{x}-2 f(x) \frac{\vec{\nabla} f(x)}{|\nabla f(x)|^{2}}, \\
& f(x)=\frac{2 f^{*}(y)}{\left|\nabla f^{*}(y)\right|^{2}}, \quad \vec{x}=\vec{y}-2 f^{*}(y) \frac{\vec{\nabla} f^{*}(y)}{\left|\nabla f^{*}(y)\right|^{2}} .
\end{aligned}
$$

Следствие. Из (6) следует тождество:

$$
H_{f} \circ H_{f *}=I_{S^{n}}=H_{f *} \circ H_{f},
$$

то есть преобразования сферы $H_{f}, H_{f *}$ - взаимно обратные и выполняется тождество:

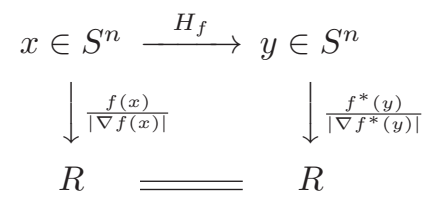


Определение 3. Одномерная секционная кривизна конформно-плоской метрики $d s^{2}=$ $\frac{d x^{2}}{f^{2}(x)}$ в $R^{n}$ задается формулой $[13,15,16]$ :

$$
K_{1 / 2}(f, x, \xi)=f \frac{d^{2} f}{d \xi^{2}}-\frac{1}{2}|\nabla f|^{2} .
$$

Здесь $\frac{d^{2} f}{d \xi^{2}}$ - вторая производная функции в точке $x \in R^{n}$ вдоль единичного вектора $\xi, \nabla f-$ градиент функции $f$ в $R^{n}$. Формула верна как в плоском случае, так и для единичной сферы, в этом случае функция $f: S^{n} \rightarrow R$ продолжается по однородности на $R^{n+1}, x \in S^{n} \subset R^{n+1}, \xi-$ единичный касательный к сфере в точке $x$ вектор, $\nabla f-$ градиент функции в $R^{n+1}$.

3. Поведение одномерной кривизны конформно-плоской метрики при преобразовании Лежандра. При аналитических выкладках, связанных с конформно-плоской метрикой, полезен метод подвижного репера Картана [14]. Обозначим через $G(n+1,1)$ многообразие базисов в $M^{n+2}$ вида:

$$
\left\{e_{1}, e_{2}, \ldots, e_{n}, Z, Z^{*}\right\} \in G(n+1,1),
$$

где $e_{1}, \ldots, e_{n}$ - пространственноподобные векторы, причем

$$
\begin{aligned}
& Z, Z^{*} \in C^{+}, \quad\left\langle Z, Z^{*}\right\rangle=-1, \\
& \left\langle Z, e_{i}\right\rangle=\left\langle Z^{*}, e_{i}\right\rangle=0, \quad i=1,2, \ldots, n .
\end{aligned}
$$

На многообразии $G(n+1,1)$ определены дифференциальные формы Маурера - Картана $\omega_{i}^{j}$ равенствами:

$$
\left(\begin{array}{c}
d e_{1} \\
\vdots \\
d e_{n} \\
d Z \\
d Z^{*}
\end{array}\right)=\left[\begin{array}{ccc}
\omega_{1}^{1} & \ldots & \omega_{1}^{n+2} \\
\vdots & \ddots & \vdots \\
\omega_{n}^{1} & \ldots & \omega_{n}^{n+2} \\
\omega_{n+1}^{1} & \ldots & \omega_{n+1}^{n+2} \\
\omega_{n+2}^{1} & \ldots & \omega_{n+2}^{n+2}
\end{array}\right] \cdot\left(\begin{array}{c}
e_{1} \\
\vdots \\
e_{n} \\
Z \\
Z^{*}
\end{array}\right)
$$

Из (8) следует

$$
\begin{aligned}
& \omega_{n+1}^{n+2}=\omega_{n+2}^{n+1}=0, \quad \omega_{n+1}^{n+1}+\omega_{n+2}^{n+2}=0, \\
& \omega_{n+1}^{k} g_{k i}-\omega_{i}^{n+2}=0, \quad \omega_{n+2}^{k} g_{k i}-\omega_{i}^{n+1}=0, \\
& \text { где } \quad g_{k i}=\left\langle e_{k}, e_{i}\right\rangle .
\end{aligned}
$$

Поверхности $F \subset C^{+}$сопоставим подмногообразие $G_{f}(n+1,1)$, выделяемое условиями:

$$
Z \in F, \quad e_{i} \in T_{z}(F), \quad i=1,2, \ldots, n .
$$

На подмногообразии $G_{f}$ дополнительно выполняется $\omega_{n+2}^{n+2}=0$. Введем обозначения $\omega_{n+1}^{i}=\omega^{i}$, $\omega_{n+2}^{i}=\omega^{* i}, i=1,2, \ldots, n$. Деривационные фор- мулы $(9)$ на $G_{f}$ примут вид

$\left(\begin{array}{c}d e_{1} \\ \vdots \\ d e_{n} \\ d Z \\ d Z^{*}\end{array}\right)=\left[\begin{array}{ccccc}\omega_{1}^{1} & \ldots & \omega_{1}^{n} & \omega_{* 1} & \omega_{1} \\ \vdots & & \vdots & \vdots & \vdots \\ \omega_{n}^{1} & \ldots & \omega_{n}^{n} & \omega_{* n} & \omega_{n} \\ \omega^{1} & \ldots & \omega^{n} & 0 & 0 \\ \omega^{* 1} & \ldots & \omega^{* n} & 0 & 0\end{array}\right]\left(\begin{array}{c}e_{1} \\ \vdots \\ e_{n} \\ Z \\ Z^{*}\end{array}\right)$.

Мы будем также пользоваться двойственным базисом $\left\{e^{1}, \ldots, e^{n}\right\}$ в пространстве $T_{z} F$. Для него имеем:

$$
\begin{aligned}
& d e^{i}=\varphi_{k}^{i} e^{k}+\omega^{i} Z^{*}+\omega^{* i} Z, \\
& d Z=\omega_{i} e^{i}, d Z^{*}=\omega_{i}^{*} e^{i},
\end{aligned}
$$

где $\varphi_{i}^{k}+\omega_{i}^{k}=0, k, i=1,2, \ldots, n$. Структурные уравнения для многообразия $G(n+1,1)$ имеют вид:

$$
d \omega_{i}^{j}=\omega_{i}^{k} \wedge \omega_{k}^{j}, \quad i, j=1, \ldots, n+2,
$$

здесь по повторяющемуся индексу $k$ берется сумма от 1 до $n+2$. Для подмногообразия $G_{f}$ отсюда получим:

$$
\begin{aligned}
& d \omega^{i}=\omega^{k} \wedge \omega_{k}^{i}, \quad d \omega^{* i}=\omega^{* k} \wedge \omega_{k}^{i}, \\
& d \omega_{i}^{j}=\omega_{i}^{k} \wedge \omega_{k}^{j}+\Omega_{i .}^{\cdot j}, \\
& \text { где } \Omega_{i .}^{\cdot j}=\omega_{i}^{*} \wedge \omega^{j}+\omega_{i} \wedge \omega^{* j}, \\
& \omega^{* i} \wedge \omega_{i}=0, \quad \text { где } k, i, j=1, \ldots, n .
\end{aligned}
$$

Из (15) следует

$$
\omega^{* i}=S^{i k} \omega_{k}, \quad S^{i k}=S^{k i}, \quad k, i=1, \ldots, n .
$$

Для форм $\Omega_{i}^{\cdot j}$ кривизны имеем представление:

$$
\Omega_{i \cdot}^{\cdot j}=\frac{1}{2} \sum_{k, l=1}^{n} R_{i \cdot k l}^{\cdot j \cdot \cdot} \omega^{k} \wedge \omega^{l},
$$

здесь $R_{i \cdot k l}^{\cdot j \cdot .}$ - тензор кривизны Римана. Тензор Риччи, скалярная кривизна, тензор Схоутена равны соответственно

$$
\begin{aligned}
& R_{i k}=\sum_{l=1}^{n} R_{i \cdot k l}^{l \cdot .}, \quad R=R_{i j} g^{i j}, \\
& S_{i k}=\frac{1}{n-2}\left\{R_{i k}-\frac{R g_{i k}}{2(n-1)}\right\} .
\end{aligned}
$$

Выясним геометрический смысл тензора $S^{i k}$. Из (15) вытекает, что формы кривизны метрики поверхности $F$ имеют вид:

$$
\Omega_{i}^{j}=d \omega_{i}^{j}-\omega_{i}^{k} \wedge \omega_{k}^{j}=\left(S_{i k} \delta_{p}^{j}+S_{p}^{j} g_{k i}\right) \omega^{k} \wedge \omega^{p} .
$$

Риманова секционная кривизна вычисляется по формуле:

$$
K(\xi \wedge \eta)=R_{i j k p} \xi^{i} \eta^{j} \xi^{k} \eta^{p}=-S_{i k} \xi^{i} \xi^{k}-S_{j p} \eta^{j} \eta^{p},
$$

где $\xi, \eta \in T_{z} F-$ ортогональные единичные векторы. 
Одномерная секционная кривизна конформноплоской метрики выражается через Риманову кривизну:

$$
K_{1 / 2}(\xi)=K\left(\xi \wedge \eta_{1}\right)+K\left(\xi \wedge \eta_{2}\right)-K\left(\eta_{1} \wedge \eta_{2}\right),
$$

где $\xi, \eta_{1}, \eta_{2} \in T_{z} F-$ ортогональные единичные векторы. Следовательно, при $n \geq 3$ одномерная секционная кривизна в направлении единичного вектора $\xi$ есть

$$
K_{1 / 2}(\xi)=-S_{i k} \xi^{i} \xi^{k}
$$

Собственные значения $k_{i}$ квадратичной формы (18), которые находятся из уравнения $\operatorname{det} \|-S_{i k}-$ $\lambda g_{i k} \|=0$ назовем главными значениями, а собственные векторы - главными направлениями одномерной кривизны.

С другой стороны, из равенства $d Z^{*}=\omega^{* i} e_{i}=$ $S^{i k} \omega_{k} e_{i}$ следует также, что матрица $\left\|S^{i k}\right\|$ задает дифференциал отображения $h_{f}$ относительно базиса $\left\{e_{1}, \ldots, e_{n}\right\}$ и двойственного базиса $\left\{e^{1}, \ldots, e^{n}\right\}$ касательного пространства $T_{Z}(F)=$ $T_{Z^{*}}\left(F^{*}\right)$. Из соображений симметрии между метриками $F$ и $F^{*}$ следует, что обратное отображение $h_{f}^{-1}: T_{Z^{*}}\left(F^{*}\right) \rightarrow T_{Z}(F)$, имеющее в качестве своей матрицы обратную к матрице $\left\|S^{i k}\right\|$, задает одномерную секционную кривизну полярной конформно-плоской метрики $F^{*} \subset C^{+}$. Матрица главных одномерных кривизн метрики $F^{*}$ диагональная и составлена из чисел, равных $1 / k_{i}$, $i=1, \ldots, n$, и главные направления кривизны на $F$ переходят в главные направления кривизны на $F^{*}$ при отображении $h_{f}$.

Следствие 1. Если конформно-плоская метрика $d s^{2}=\frac{d x^{2}}{f^{2}(x)}$ имеет положительную одномерную кривизну, то $H_{f}$ диффеоморфизм (3) сферы $S^{n}$ и полярная конформно-плоская метрика $d s^{* 2}=\frac{d y^{2}}{f^{* 2}(y)}$ также имеет положительную одномерную кривизну.

Замечание. Кривизна Риччи и одномерная секционная кривизна конформно-плоской метрики $F$ выражаются друг через друга $(n \geq 3)$ :

$$
\begin{aligned}
& R_{i k}=(n-2) S_{i k}+g_{i k} S, \quad S=S_{i k} g^{i k}, \\
& S_{i k}=\frac{1}{n-2}\left\{R_{i k}-\frac{R g_{i k}}{2(n-1)}\right\}, \quad R=R_{i k} g^{i k} .
\end{aligned}
$$

Аналогично для полярной поверхности $F^{*}$ :

$$
\begin{aligned}
& \tilde{R}_{i k}=(n-2) \tilde{S}_{i k}+\tilde{g}_{i k} \tilde{S}, \quad \tilde{S}=\tilde{S}_{i k} \tilde{g}^{i k}, \\
& \tilde{S}_{i k}=\frac{1}{n-2}\left\{\tilde{R}_{i k}-\frac{\tilde{R} \tilde{g}_{i k}}{2(n-1)}\right\}, \quad \tilde{R}=\tilde{R}_{i k} \tilde{g}^{i k},
\end{aligned}
$$

где $g_{i k}=\delta_{i k}, \tilde{g}_{i k}=\delta^{p s} S_{i p} S_{j s},\left\|\tilde{S}_{i}^{k}\right\|=\left\|S_{i}^{k}\right\|^{-1}$.

Пример. Пусть конформно-Плоская метрика в $R^{3}$ задана функцией $f\left(x_{1}, x_{2}, x_{3}\right)=1+x_{1}^{2}+x_{2}^{2}$, тогда

$$
\begin{aligned}
f^{*}\left(y_{1}, y_{2}, y_{3}\right) & =\frac{1}{2}\left(y_{1}^{2}+y_{2}^{2}+1\right) \\
y_{1} & =-\frac{x_{1}}{x_{1}^{2}+x_{2}^{2}}, \\
y_{2} & =-\frac{x_{2}}{x_{1}^{2}+x_{2}^{2}}, \\
y_{3} & =x_{3}
\end{aligned}
$$

Выражая из двух последних уравнений $x_{1}, x_{2}, x_{3}$ и подставляя в первое уравнение, получим

$$
f^{*}\left(y_{1}, y_{2}, y_{3}\right)=\frac{1}{2}\left(y_{1}^{2}+y_{2}^{2}+1\right) \text {. }
$$

Главные значения одномерной секционной кривизны метрик $d s^{2}, d s^{* 2}$ равны соответственно:

$$
\begin{aligned}
& K_{1 / 2}(f)=\left(\begin{array}{ccc}
2 & 0 & 0 \\
0 & 2 & 0 \\
0 & 0 & -2\left(x_{1}^{2}+x_{2}^{2}\right)
\end{array}\right), \\
& K_{1 / 2}\left(f^{*}\right)=\left(\begin{array}{ccc}
\frac{1}{2} & 0 & 0 \\
0 & \frac{1}{2} & 0 \\
0 & 0 & \frac{1}{2}\left(-y_{1}^{2}-y_{2}^{2}\right)
\end{array}\right) .
\end{aligned}
$$

\section{Библиографический список}

1. Brinkmann H.W. On Riemann spaces conformal to Euclidean spaces // Proc. Nat. Acad. Sci. USA 9 (1923), 1-3.

2. Kuiper N.H. On conformally-flat spaces in large // Ann. of Math. - (2) 1949. - V. 50.

3. Kuiper N.H. On compact conformally Euclidean spaces of dimention $>2 / /$ Ann. of Math. - (2) 1950. - V. 52.

4. Кантор Б.Е., Франгулов С.А. Об изометричном погружении двумерных римановых многообразий в псевдоевклидово пространство Мат. заметки. - 1984. - Т. 36, № 3.
5. Славский В.В. Конформно плоские метрики ограниченной кривизны на $n$-мерной сфере. Исследования по геометрии «в целом» и математическому анализу. - Новосибирск, 1987. - Т. 9.

6. Udo Hertrich-Jeromin. Introduction to Mobius Differential Geometry. London mathematical society lecture note series. - Cambridge University Press, 2003.

7. Решетняк Ю.Г. Теоремы устойчивости в геометрии и анализе. - Новосибирск, 1996.

8. Топоногов В.А. Дифференциальная геометрия кривых и поверхностей. - М., 2012. 
9. Slavskii V.V. Conformally flat metrics and the geometry of the pseudo-Euclidean space Siberian Math. J. - 35 (1994).—№ 3.

10. Славский В.В. Оценка коэффициента квазиконформности области через кривизну квазигиперболической метрики // Сиб. мат. журн. 1999. - Т. 40, № 4.

11. Славский В.В. Конформно-плоские метрики и псевдоевклидово пространство : автореф. ... дисс. докт. матем. наук. - Новосибирск, 2000.

12. Славский В.В. Геометрический подход в многомерной теории потенциала // Труды по анализу и геометрии. - Новосибирск, 2000.

13. Балащенко В.В., Никоноров Ю.Г., Родионов Е.Д., Славский В.В. Однородные пространства: теория и приложения : монография. Ханты-Мансийск, 2008.
14. Картан Э. Риманова геометрия в ортогональном репере. - М., 1960.

15. Родионов Е.Д., Славский В.В. Одномерная секционная кривизна римановых многообразий // Доклады академии наук. - 2002. - Т. 387, № 4 .

16. Nikonorov Yu.G., Rodionov E.D., Slavskii V.V. Geometry of homogeneoues Riemannian manifolds // Journal of Mathematical Scieces. 2007. - V. 146, № 6 .

17. Kurkina M.V., Rodionov E.D. and Slavskii V.V. Conformally Convex Functions and Conformally Flat Metrics of Nonnegative Curvature // Doklady Mathematics. - 2015. V. 91, № 3 .

18. Родионов Е.Д., Славский В.В. Полярное преобразование конформно-плоских метрик // Математические труды. - 2017. - Т. 20, № 2. 